\title{
Computer Numerically Controlled Machine to Draw text on Ardunio-based Acrylic Board
}

\author{
M. Miftakul Amin, Hartati Deviana, Raksi Andika, Phong Thanh Nguyen, Wahidah Hashim, \\ Andino Maseleno
}

\begin{abstract}
This paper presents Computer Numerically Controlled (CNC) machine to draw text on Arduino based acrylic board. The principle working of Computer Numerical Control (CNC) is a machine that is used for automatic control in the industrial world. It must more accurate, precise, flexible and suitale for production. CNC can reduce operator intervention during its operation. Design of computer numerically controlled machines for drawing text on Arduino-based acrilic boards is a tool used to draw text directly to an acrilic board based on the coordinates obtained from inkscape software. This tool consists of three translating axes, namely the $X, Y$, and $Z$ axes. Each axis will move according to each coordinate obtained from the inkscape software and then sent to the microcontroller. This tool is only focused to draw the text is expected to not only draw the text, but also can draw another shape.
\end{abstract}

IndexTerms:drawing design; acrylic board; computer numerically controlled; Arduino UNO; CNC machine

\section{INTRODUCTION}

\section{Background}

Computer numerical controlled (CNC) machine and robotic systems has become modern manufacturing industry [1] and educational institutions and laboratories [2]. The most common activity performed in today's technologically advanced world is writing, drawing, etc. which is performed manually and time consuming [3]. By using $\mathrm{CNC}$, the machining track can be directly generated, so the motion control can be achieved [4]. Along with the era development, the technology is growing rapidly, one of them is the development of robotics and electronics, for example, the process of making text drawings on acrylic board, in making text drawing design on acrylic board usually print the first design drawing using the printer, then design the text drawing and paste to the acrylic board, the process spends a lot of time and the result can not match as desired and less efficient.For that reason the author will realize a tool that can create text drawing designon acrylic board automatically by Computer Numerically Controlled machine with computer which is run by a program to create text drawing design on acrylic board.

Revised Manuscript Received on June 22, 2019.

M. Miftakul Amin, Politeknik Negeri Sriwijaya, Palembang, Indonesia. Hartati Deviana, Politeknik Negeri Sriwijaya, Palembang, Indonesia. Raksi Andika, Politeknik Negeri Sriwijaya, Palembang, Indonesia.

Phong Thanh Nguyen, Department of Project Management, Ho Chi Minh City Open University (HCMCOU), Vietnam.

Wahidah Hashim, Institute of Informatics and Computing Energy, Universiti Tenaga Nasional, Malaysia.

Andino Maseleno, Institute of Informatics and Computing Energy, Universiti Tenaga Nasional, Malaysia.

Corresponding author e-mail: andimaseleno@gmail.com

This machine can run according to the text drawing design we want. Users just onlydesigntext or text drawing with computer devices, so it can save time and become efficient.

This research developed CNC machine technology, while this tool has been made in the European country, but the author designed a CNC machine that is different from the one on the market, for example in terms of mechanical and control system, for control system uses arduinouno microcontroller.

\section{Problem Formulation}

The problem faced is how to create a text image design on the acrylic board without printing the text image design and attach it to acrylic board using $\mathrm{CNC}$ machine.

\section{Problem Limitation}

In writing this research to be more focused and no elaboration of the problem, the authors simplify and direct the research so as not to deviate of what is being observed, The problem limitations are as follows:

1. The type of ink is permanent or not permanent and only one colour

2. Creating design using inkscape software and saved to be g-code.

3. Software for processing g-code runs using CAMotics.

4. Software used to process g-code and run machine is Processing

5. The size of paper used is $20 \times 29 \mathrm{~cm}$.

6. Focused to draw text

\section{Purpose}

The purpose to be achieved in this tool designing was to make numerically controlled computer machine to draw text on acrylic board.

\section{Benefit}

Therefore the benefits of making this tool are

1. Minimize the error rate in making the design on the acrylic board.

2. Ease the design creation on the acrylic board

\section{LITERATURE REVIEW}

\section{CNC Machine}

Machine CNC is a machine controlled by a computer with command data code numbers, letters, and symbols, according to standard ISO [5]. The beginning of CNC (Computer Numerically Controlled) machine birth that was originated developed by John Pearseon in 1952 from the Massachusetts

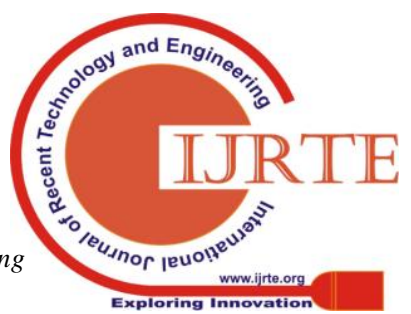


Institute of Technology, on behalf of the United States Air Force. Originally the project was devoted to make complex specialized workpieces. Originally a CNC machine device required a high cost and large volume control unit.

In 1973, CNC machine was very expensive so that few companies had the courage to pioneer investment in this technology. From 1975, the production of CNC machines began to grow rapidly. This development was driven by the development of microprocessors, so that the volume of control units can be more concise.

CNC machine is machine controlled by computer using numerical language (data of command with number code, letter and symbol) according to ISO standard. This CNC machine uses software such as Arduino, Processing, Camotic, and Inkscape. As for some hardwares used namely Uno Arduino, Adafruit Motor Shield, Nema 16 Motor Stepping and the mechanics. The design process must be done correctly, from material selection to mechanical, machine design, software installation, synchronization until testing, so that machine can work in accordance with the program that has been made and produce the expected product.

\section{Arduino Uno Microcontroller}

Microcontroller is a chip that serves as an electronic circuit controller and generally it can store the program therein. Microcontroller generally consist of CPU (Central Processing Unit), memory, certain I / O and support units such as Analog-to-Digital Converter (ADC) which is already integrated in it [6].

Arduino is an electronic kit or an open source electronic circuit board in which there is a main component that is a microcontroller chip with AVR type from Atmel company. Chip or IC (integrated circuit) can be programmed using computer. The purpose of embedding the program on a microcontroller is the electronic circuit can read the input, process the input and then produce the expected output. So the microcontroller serves as the 'brain' that controls the input, process and output of an electronic circuit.

In the Arduino Block Diagram there are several parts namely:

a. Universal Asynchronous Receiver / Transmitter (UART) is the interface used for serial communication such as RS-232, RS-422 and RS-485.

b. $2 \mathrm{~KB}$ RAM in working memory is volatile (lost when power is off), used by variables within the program.

c. 32KB RAM flash memory is non-volatile, used to store program loaded from the computer. In addition to programs, flash memory also keeps the bootloader.

d. Bootloader is a small initiation program, run by CPU when power is turned on. After bootloader is finished, next program in RAM will be executed.

e. $1 \mathrm{~KB}$ EEPROM is non-volatile, used to store data that should not be lost when power is turned off. Not used on Arduino board (red: but accessible / programmed by user and used as needed).

f. Central Processing Unit (CPU), part of microcontroller to run every instruction from the program.

g. Input / output ports, pins to receive digital or analog data, and output digital or analog data (output).

For more details about Architecture Uno Arduino can be seen in figure 1.

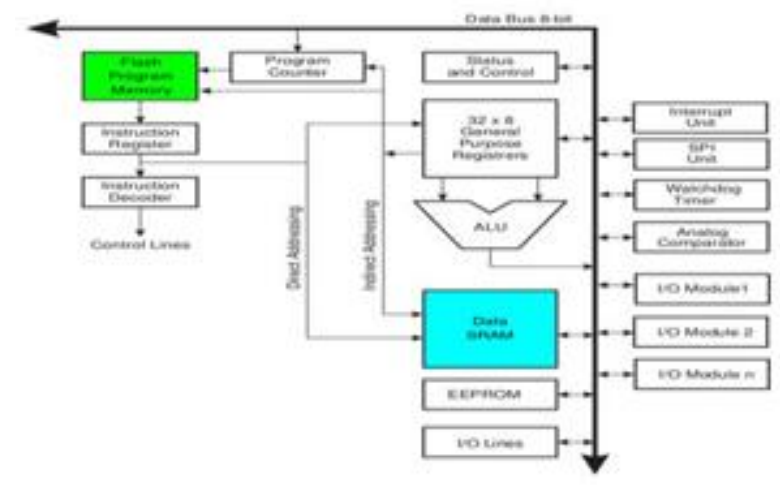

Fig. 1 Atmega 328 Architecture

\section{Atmega328 Pin Configuration}

The following is the ATmega328 configuration, it can be seen in figure 2 .

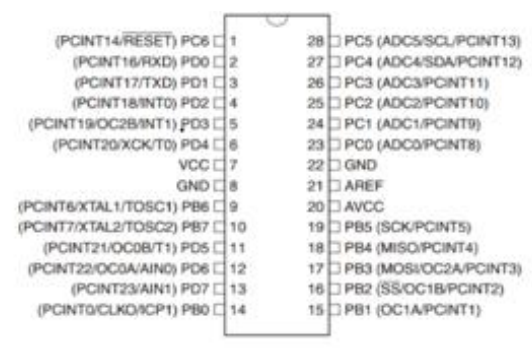

Fig. 2 Atmega 328 Pin Configuration

ATMega328 had 28 Pins, each pin has different functions as both port and other functions. The following will explain the function of each ATmega8 leg as follows:

- VCC is a digital voltage supply.

- GND is the ground for all components that requires grounding.

- Port B (PB7-PB0) Inside Port B there are XTAL1, XTAL2, TOSC1, TOSC2. The number of Port B is 8 pins, starting from pin B.0 to B.7. Each pin can be used as input or output. Port B is an 8-bit bidirectional I / O with an internal pull-up resistor. As an input, the pins are on port $\mathrm{B}$ that is externally lowered, it will put out the current if the pull-up resistor is activated. Special PB6 can be used as a crystal input (inverting oscillator amplifier) and input to an internal clock circuit, depending on the Fuse bit setting used to select the clock source. As for PB7 it can be used as a crystal output (output oscillator amplifier) depending on the Fuse bit setting used to select the clock source.

If the clock source is selected from the internal oscillator, PB7 and PB6 can be used as I / O or if using Asyncronous Timer / Counter2 then PB6 and PB7 (TOSC2 and TOSC1) are used for timer input channel.

- Port C (PC5-PC0), Port C is a 7-bit bi-directional I / O port which inside each pin is a pull-up resistor. The number of pins is only 7 pieces starting from pin C.0 to pin C.6. As output / output port $\mathrm{C}$ has the same characteristics in terms of absorbing current (sink) or issuing current (source).

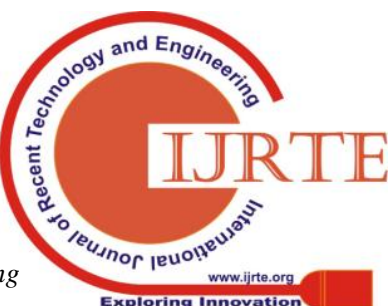


- Fuse is programmed, then PC6 will function as I / O pin. This pin has different characteristics with the pins found on other C ports. However if RSTDISBL Fuse is not programmed, then this pin will serve as reset input. And if the voltage level coming into this pin is low and the pulse is shorter than the minimum pulsa, it will produce a reset condition even if the clock is not working.

- Port D (PD7-PD0) Port D is an 8-bit bi-directional I/ O with an internal pull-up resistor. The function of this port is same as the other ports. But in this port there are no other utilities. In this port only serves as input and output only or commonly called the I / O.

- Avcc, This pin functions as a supply voltage for ADC. For this pin must be connected separately with VCC because this pin is used for analog only. Even if ADC on AVR is not used it is recommended to connect it separately with VCC. If ADC is used, then AVcc must be connected to $\mathrm{VCC}$ via low pass filter.

- AREF is a reference pin if it uses ADC

Uno Arduino can be enabled via USB connection or with external power supply. The power source is automatically selected. External (non-USB) power can come from either an AC-DC adapter or a battery. This adapter can be connected by connecting a $2.1 \mathrm{~mm}$ center-positive plug into the power jack. The lead from the battery can be inserted into the Gnd and Vin pin headers of the Power connector. The board can operate on a power supply of $6-20$ volts. If it is given with less than $7 \mathrm{~V}$, however, $5 \mathrm{~V}$ pins can supply less than 5 volts and the board may be unstable. If it uses more than $12 \mathrm{~V}$, the voltage regulator can heat and damage the board. The recommended range is $7-12$ volts.

The power supply pins are as follows:

- VIN. Input voltage to Arduino board when using external power source (as opposed to 5 volts from USB connection or other set power source). You can supply voltage through this pin, or, if you supply voltage through the power outlet then you can access it through this pin.

- $5 \mathrm{~V}$. The power supply is used for microcontroller power and other components on the board. This can happen either from VIN via the onboard regulator, or provided by USB.

- 3,3 volt supply volts produced by on-board regulators. The maximum draw current is $50 \mathrm{~mA}$.

- GND

\section{Memory}

The ATmega328 has $32 \mathrm{~KB}$ with $0.5 \mathrm{~KB}$ used for loading files. It also has $2 \mathrm{~KB}$ of SRAM and $1 \mathrm{~KB}$ of EEPROM.Input and Output

Each of the 14 digital pins on Uno can be used as input or output, using pinMode (), digitalWrite (), and digitalRead () functions. They operate at 5 volts. Each pin can provide or receive a maximum of $40 \mathrm{~mA}$ and has an internal pull-up resistor of 20-50 K_.

In addition, some pins have special functions:

- Serial: 0 (RX) and 1 (TX). It is used to receive (RX) and send (TX) TTL serial data. This pin is connected to the corresponding pin of the USB-to-Serial TTL ATmega8U2 chip.

- External Interruptions: 2 and 3. This pin can be configured to trigger interruptions at low values, rising or falling edges, or changing values. See attachInterrupt () function for details.

- PWM: 3, 5, 6, 9, 10, and 11. Provide 8-bit PWM output with analogWrite () function.

- SPI: 10 (SS), 11 (mosi), 12 (MISO), 13 (SCK). This pin supports SPI communication using SPI library.

- There is a built-in LED connected to the digital pin 13 When the pin is a HIGH value, the LED lights up, when the pin is LOW, it's off.

Uno has 6 analog inputs, labeled A0 through A5, each of them provides 10 bits resolution that is 1024 different values. By default system measures from ground to 5 volts.

- TWI: 4 or SDA pins and A5 or SCL pins. Supports TWI communication

- Aref. Voltage reference for analog input. Used by analogReference ().

- Reset.

See the mapping between the Arduino pin and ATmega328 port. Mapping for ATmega8, 168 and 328 is identical.

\section{Communication}

Uno Arduino has a number of facilities to communicate with a computer, another Arduino, or another microcontroller. ATmega328 provides UART TTL (5V) serial communication, which is available on digital pins 0 (RX) and 1 (TX). An ATmega16U2 on this board channel is serial communication via USB and appears as a virtual com port for software on the computer. The Arduino firmware uses the standard COM USB driver, and no external drivers are needed. However, on Windows, the file.Inf needed. The Arduino software includes a serial monitor that allows simple data to be sent to the Arduino board. RX and TX The LED on the board will blink when data is being sent via a USB-to-serial chip and a USB connection to the computer. ATmega328 also supports I2C communication (TWI) and SPI. This function is used to communicate interface on the system.

\section{Programming}

The Uno Arduino can be programmed with the Arduino. software. Select Uno Arduino from tool then adjust it to the microcontroller used. The ATmega328 on Uno Arduino has a bootloader that allows you to upload new programs without using an external hardware programmer. It communicates using protocols from $\mathrm{C}$ language. The system can use Atmel FLIP software (Windows) or DFU programmers (Mac OS X and Linux) to load new firmware. Or you can use an ISP header with an external programmer.

\section{(Arduino IDE) Sofware}

IDE is abbreviation for Integrated Developtment Enviroenment. Arduino uses its own programming language that resembles $\mathrm{C}$ language. In the Arduino microcontroller a program called Bootlader has been entered which functions as an intermediary between the Arduino compiler and the microcontroller. 
Arduino IDE is made from JAVA programming language. The Arduino IDE also comes with a $\mathrm{C} / \mathrm{C}++$ library that is usually called Wiring which makes input and output operations easier. This Arduino IDE was developed from Processing software which was overhauled into an Arduino IDE specifically for programming with Arduino.

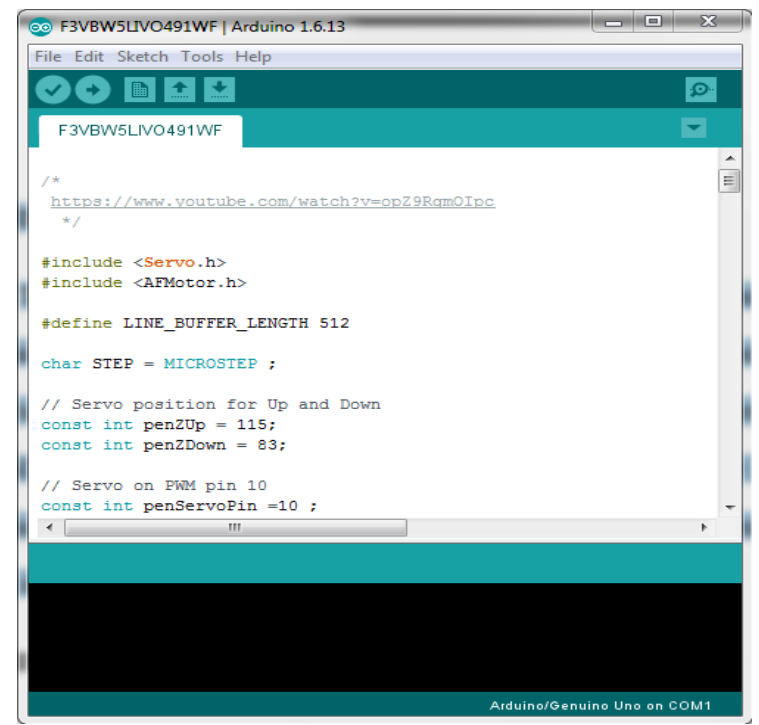

Fig. 3 Arduino Program Display

\section{Automatic Software Reset}

Uno Arduino reset button is designed to run programs stored in the microcontroller from scratch. The reset button is connected to Atmega328 via a 100nf capacitor. After the reset button is pressed long enough to reset the chip, the Arduino IDE software can also serve to upload the program by simply pressing the upload button in IDE Arduino software.

\section{Adafruit Motor Shield}

Adafruit Motor Shield is an arduino shield that is easy to use for making robotic applications. Because this shield can run four pieces of motor and two servo at once. Physical form of driver adafruit motor shield can be seen in figure 4 .

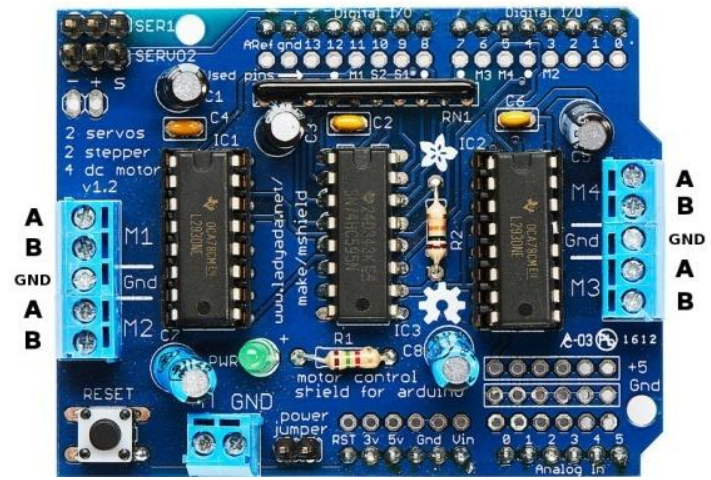

Fig. 4Adafruit Motor Shield

The full specification of this shield is as follows.

a. 2 connector for $5 \mathrm{~V}$ Servo.

b. Can run 4 motor DC atau 2 stepper motors atau 2 Servos

c. Can run 4 motor bi-directional DC with selection speed 8-bit.

d. Run 2 stepper motors (unipolar atau bipolar) with single coil or double coil. e. 4 H-Bridges: each bridge provides 0.6A (1.2A when culimanting) with thermal proctection, it can run $4.5 \mathrm{~V}$ until 36V DC motor.

f. Reset button

g. Two External power connectors

Figure 5 shows IC L293D.

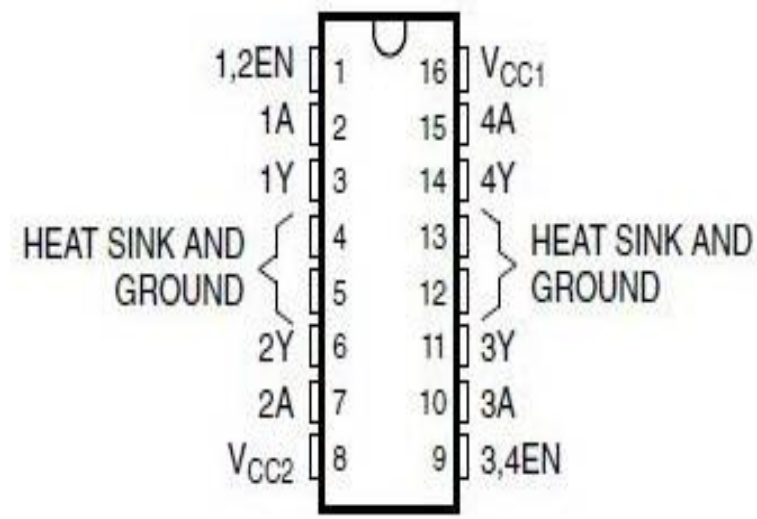

Fig. 5 Ic L293D

\section{Stepper motor}

Stepper motor is a motor that is used as a drive or player. The working principle of a stepper motor is similar to a DC motor, equally stacked with a DC voltage to obtain a magnetic field. When a DC motor has a fixed magnet on the stator, the stepper motor has a fixed magnet on the rotor. The stepper motor is expressed by specification: "how many phases", "how many degrees perstep", "how many volt supply voltages for each winding" and "how many amperes / miliampere currents are needed for each winding". The stepper motor can not move itself, but moves per-step according to its specifications, and moving from one step to the next step takes time, and produces great torque at low speeds. The stepper motor also has other characteristics, namely retaining torque, which allows it to hold its position. This is very useful for applications where a system requires a start and stop state [7]. The inside of a stepper motor can be seen in figure 6 .

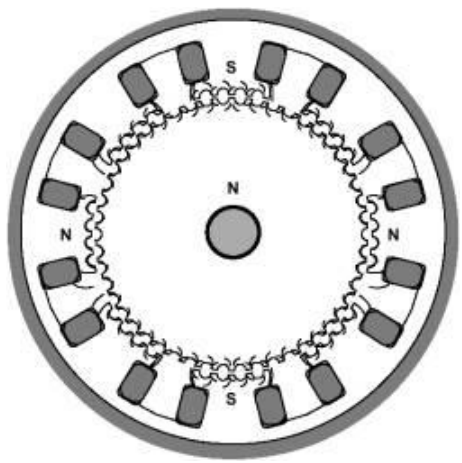

Fig. 6 Stepper motor

The stepper motor does not respond to the clock signal and has several turns where the windings must be fed (voltage) first in a certain order in order to rotate. Reversing the sequence of voltage assignments will cause the rotation of the stepper motor to reverse direction. If the control signal is not sent according to the command, the stepper motors will not rotate properly,

\section{Published By:}


it may only vibrate and not move. To control the stepper motor, it is used a circuit of drivers that handle current and voltage requirements.

\section{Stepper motor Charactheristic}

The charactheristics of stepper motor according to Trianto are as follows :

a. Each motor stepper has the average voltage written on each unit or listed on the datasheet of each stepper motors. This average voltage must be considered carefully because if it exceeds the average voltage this will cause heat which causes the rotation performance not be maximal or even the stepper motors will be damaged by itself.

b. Resistance per coil is another characteristic of stepper motors. This resistance will determine the current flowing, but it will also affect the maximum torque and speed and the stepper motors.

c. Degree per step is the most important factor in the selection of stepper motor according to the application. Each motor stepper has its own specifications, including: $0.72^{\circ}$ per step, $1.8^{\circ}$ per step, $3.6^{\circ}$ per step, $7.5^{\circ}$ per step, $15^{\circ}$ per step, and even $90^{\circ}$ per step. In operation we can use 2 principles, namely full step or half step. With full step it means the stepper motor rotates according to the degree specification per step, while the half step means the stepper motors rotates half a degree per step of the specification of the stepper motors.

\section{Type of Stepper motor}

Stepper motors is divided into two broad categories: permanent magnet and variable reluctance. The permanent magnet type is divided into two, namely unipolar and bipolar stepper motors. Stepper motors unipolar is easy to be controlled by using $\mathrm{n}$ counter circuit Stepper motors unipolar has special characteristic namely there is center tapped winding and one winding as common. Common threads will supply the center-tapped voltage and as ground is the driver circuit. Unipolar stepper motors can be identified by knowing the center-tapped winding. The number of phase and stepper motors is twice and the number of coils. Generally on unipolar stepper motors there are two coils. Stepper motors diagram can be seen in figure 7 .

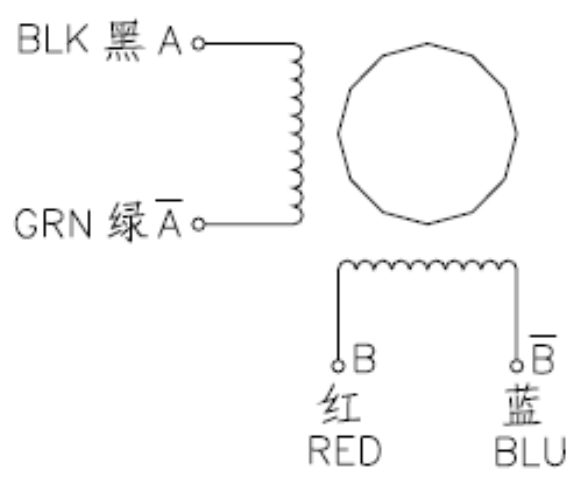

Fig. 7 Stepper motors 4 Wire Diagram Stepper motor Working Principal

In principle, there are two types of working for unipolar stepper motors, namely the full-step and half-steps described in table 1 and table 2 .
Table. 1 FullStep Method

\begin{tabular}{|c|c|c|c|c|c|c|c|c|}
\hline \multicolumn{9}{|c|}{ FULLSTEP } \\
\hline \multicolumn{9}{|c|}{ Voltage given winding } \\
\hline \multirow[t]{2}{*}{ No } & \multicolumn{4}{|c|}{$\begin{array}{l}\text { Rotate direction } \\
\text { clockwise }\end{array}$} & \multicolumn{4}{|c|}{$\begin{array}{c}\text { Rotate direction agains } \\
\text { clockwise }\end{array}$} \\
\hline & L3 & L2 & L1 & L0 & L3 & $\mathrm{L} 2$ & $\mathrm{~L} 1$ & L0 \\
\hline 1 & 1 & 0 & 0 & 0 & 0 & 0 & 0 & 1 \\
\hline 2 & 0 & 1 & 0 & 0 & 0 & 0 & 1 & 0 \\
\hline 3 & 0 & 0 & 1 & 0 & 0 & 1 & 0 & 0 \\
\hline 4 & 0 & 0 & 0 & 1 & 1 & 0 & 0 & 0 \\
\hline
\end{tabular}

At full step, a point on a magnetic pole in the rotor will get the pull of the stator magnetic field in the same winding after step 4., and the next can be given again starting from step 1. Each step, the rotor rotate direction clockwise or against clockwise as big as the specification of degree per step and stepper motor. Each step only pulls a pole. The '1' voltage is indicating logic in the Transistor Transistor (TTL) level. The actual voltage is set by the stepper motor specifications used, for example by using a buffer.

\section{Table.3 HalfStep Method}

\begin{tabular}{|c|c|c|c|c|c|c|c|c|}
\hline \multicolumn{9}{|c|}{ HALFSTEP } \\
\hline \multicolumn{9}{|c|}{ Voltage given to winding } \\
\hline \multirow[t]{2}{*}{ No } & \multicolumn{4}{|c|}{$\begin{array}{l}\text { Rotate direction } \\
\text { clockwise }\end{array}$} & \multicolumn{4}{|c|}{$\begin{array}{l}\text { Rotate direction against } \\
\text { clockwise }\end{array}$} \\
\hline & L3 & $\mathrm{L} 2$ & L1 & L0 & L3 & L2 & L1 & L0 \\
\hline 1 & 1 & 0 & 0 & 0 & 0 & 0 & 0 & 1 \\
\hline 2 & 1 & 1 & 0 & 0 & 0 & 0 & 1 & 1 \\
\hline 3 & 0 & 1 & 0 & 0 & 0 & 0 & 1 & 0 \\
\hline 4 & 0 & 1 & 1 & 0 & 1 & 1 & 1 & 0 \\
\hline 5 & 0 & 0 & 1 & 0 & 1 & 1 & 0 & 0 \\
\hline 6 & 0 & 0 & 1 & 1 & 1 & 1 & 0 & 0 \\
\hline 7 & 0 & 0 & 0 & 1 & 0 & 0 & 0 & 0 \\
\hline 8 & 1 & 0 & 0 & 1 & 0 & 0 & 0 & 1 \\
\hline
\end{tabular}

For half step, each magnetic pole on the rotor will get back the same pull and winding magnetic field after the next step 8. starting from step 1. Each step of the rotor position changes by half a degree and the degree specification per step stepper motor.

Unlike with the unipolar stepper motor, bipolar stepper motor is very difficult to be controlled. This type of stepper motor requires a complex driver circuit. The advantages of bipolar stepper motors are their large size and can produce large torque rather than unipolar stepper motors. Stepper bipolar motor is designed with separate winding that will be supplied and bidirectional (polarity must be reversed during ripping). Bipolar stepper motor uses the same logic as unipolar stepper motor which is only ' 0 ' and ' 1 ' to respond to the coil

\section{Servo Motor}

Servo motor is device or actuator (motor) designed with a closed loop feedback control system (servo), so that it can be set-up or adjusted to determine and ensure the angle position of the motor output shaft. There are bacically 2 types of servomotor: AC and DC servomotor [8].

Servo motor is a type of motor that has three cables. Each isused as a power supply, ground, and control. 
The control cable is used to determine the motor to rotate the rotor to a certain position. Normally, the rotor only rotates up to $200^{\circ}$. However, some rotors are capable of rotating by $360^{\circ}$.

Servo motors is commonly used to move a robotic arm or rotate an analogue measuring instrument, or as additional accessories for the Arduino project.

Usually, the servo motor is equipped with three red, orange and brown cables.

a. Red cable is connected to 5 volt power supply

b. Orange cable is connected to digital pin which supports PWM.

c. Brown cable is connected to ground

\section{Servomotor working principal}

Servo motor is controlled by providing pulse width modulation (PWM) signals via control cables. The width of provided control signal pulse will determine the angular position of the rotation of the servo motor shaft. The servo motor component can be seen in figure 8 .

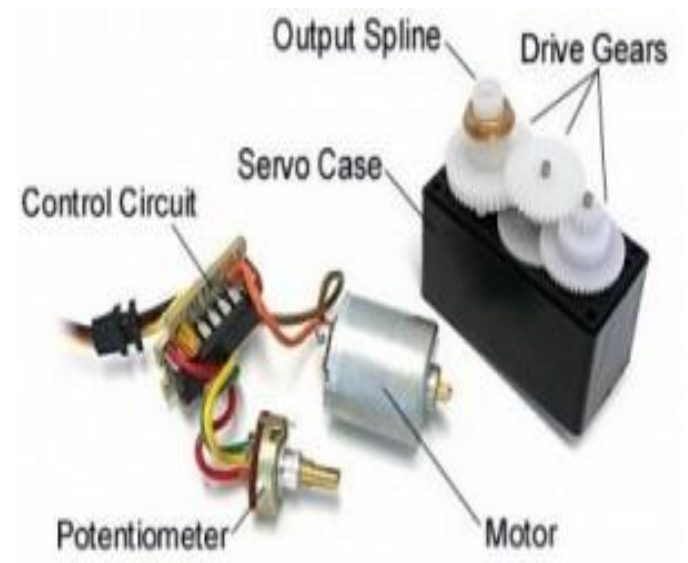

Fig. 8 Servo motor component

For example, the pulse width with a time of $1.5 \mathrm{~ms}$ (milli seconds) will rotate the servo motor shaft to a $90^{\circ}$ angle position. If the pulse is shorter than $1.5 \mathrm{~ms}$, it will rotate toward position $0^{0}$ or left (counterclockwise), whereas if the pulse given is longer than $1.5 \mathrm{~ms}$, the servo axis of the motor will rotate toward the position $180^{\circ}$ or to the right (clockwise), the servo motor block diagram can be seen in figure 9 .

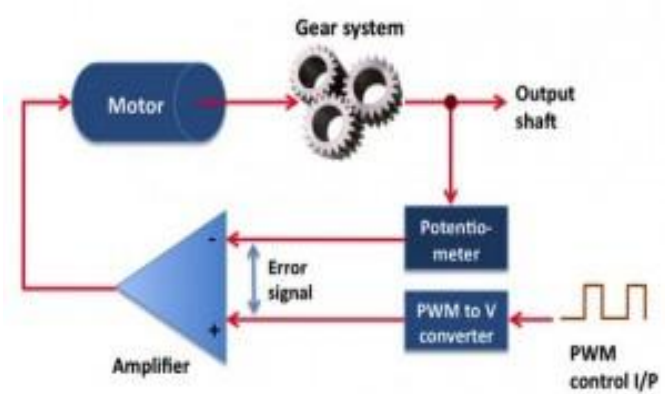

Fig. 9 .Servo motor Diagaram block

\section{Serial Communication}

Serial communication is a communication that transmits data per-bit sequentially and interchangeably. This communication has an advantage that requires only one path and a few wires compared to parallel communication. In principal, serial principle communication is a communication where data transmission is done per bit slower than parallel communication, or in other words serial communication is one method of data communication where only one bit of data is sent through a strand of cable at a certain time. Basically serial communication is a special case of parallel communication with a value of $\mathrm{n}=1$, or in other words is a form of parallel communication with only one number of cables and only sending one bit of data simultaneously. This can be paired with real parallel communication where $\mathrm{n}$-bit data is sent simultaneously, with a common value of $8 \leq \mathrm{n} \leq$ 128.

Serial communication has two types, asynchronous serial and synchronous serial. Synchronous serial is a communication where there is only one party (sender or receiver) that produces a clock and sends the clock together with the data. Examples of serial synchronous usage is found on keyboard data transmission. Asynchronous serial is a communication where both parties (sender and receiver) each produce a clock but only the data that are transmitted, without clock. So that the data sent are the same as the data received, then the two clock frequencies must be the same and there must be synchronization. After synchronization, the sender will send the data according to the sender's and receiver's clock frequency will read the data according to the receiver's clock frequency. An example of using asynchronous serial is Universal Asynchronous Receiver Transmitter (UART) which is used on computer serial port (COM). UART is an integrated circuit which plays the most important role in serial communication [9].

The interface serial channel is more complex or difficult than the parallel channel interface, because:

a. In terms of hardware: the process of converting parallel data into serial or vice versa using an additional device called UART (Universal Asynchronous Receiver / Transmitte) and

b. In terms of software: more registers are used or involved

But on the other side of the channel interface serial, it offers many advantages compared in parallel, among others:

a. Cable for serial communication can be longer than parallel; data in serial communication is sent to logic ' 1 ' as voltage $-3 \mathrm{~s} / \mathrm{d}-25$ volts and for logic ' 0 ' as voltage $+3 \mathrm{~s} /$ $\mathrm{d}+25$ volts, thus the voltage in serial communication has a voltage swing maximum of 50 volts, while in parallel communication only 5 volts. This causes interference to long cables more easily overcome than in parallel.

b. The number of serial cables is less; You can connect two remote computer devices with only 3 cables for the null modem configuration, ie TXD (send line), RXD (receive line) and Ground, imagine if the parallel technique is used there will be 20 - 25 cables. But on each computer with serial communication should be paid "cost" of serial interface which is rather expensive.

c. The number of devices today (palmtop, organizer, hand phone and others) uses infrared technology for data communication, in this case the data transmission is done serially. IrDA-1 (the first infrared specification) is able to transmit data at a rate of $115.2 \mathrm{kbps}$ and the Serial 2 Communication Concept is assisted with a UART device, The pulse length is reduced to $3 / 16$ from the RS-232 standard to save power.

\section{Published By:}


d. For embedded system technology, many microcontrollers are equipped with serial communication (both RISC and CISC series) or Serial Communication Interface (SCI); with the SCI integrated at $1 \mathrm{C}$ the microcontroller will reduce the number of output pins, so it only takes 2 main pins TxD and RxD (outside the ground reference).

\section{Serial Arduino Communication}

Arduino serial communication is Communication between Uno Arduino and Computer, it can be done via serial port (via USB). In this case, Uno Arduino can not only read data from a computer on a serial port, but can also send data to a computer [10]. So that the communication is two-way.

The Arduino IDE adjusts the facility to carry out two-way communication via a serial monitor. By using this facility, data can be sent to Uno Arduino and vice versa it can read posts from Uno Arduino. Of course, it is possible to control Uno Arduino through a computer and monitor what is happening in Uno Arduino. For example, when sending a signal to turn on the lamp or monitor the temperature detected by the temperature sensor in the Serial Monitor.

Command Type of Arduino Communication Serial

a. Begin serial; () : useful for determining the speed of sending and receiving data via serial port. The commonly used speed is 9600 bits per second ( $9600 \mathrm{bps})$. However, speed up to 115,200 is supported by Uno Arduino. Example: begin serial (9600).

b. End serial (): used for stopping communication serial

c. Available serial : useful for generating the number of bytes in the unreadable serial port. If the serial port is empty, this function can produce a zero value.

d. Read serial () :

e. useful for reading one byte of data contained in the serial port. After read serial (), the number of data in the serial port is reduced by one. To read all data, the following commands are required:int datawhile $($ Serial.available ()$)\{$ data $=$ Serial.read ()$;\}$

f. Print(data) serial : function to send data to serial port. If format argument is included, the data submitted will match that format. In this case, the format used can be:

g. DEX : decimal format or basis 10

h. HEX : hexadicimal atau basis 16

i. OCT : octabe format atau basis 8

j. BIN : biner format atau basis 2

$\mathrm{k}$. If the argument is only one, the result is in string form. For example : Print serial(65); //hasilnilaitertampil 65

1. Print serial(65,DEC) //displayed result value 65

m. Print serial $(65, \mathrm{HEX})$; //displayed result value 41

n. Print serial $(65$, OCT) //displayed result vale 101

o. Print serial(65, BIN) //displayed result value 1000001

p. Flush serial() : It has the function to empty the reading data that is placed in the buffer.

q. parseFloat serial() bergunauntukbilangantitikmengambangatau real.

r. serialln(data) print : has almost the same function as print serial, which gives the effect of moving the next line.

s. parseln() serial : to generate integer value .

\section{DESIGN}

\section{Designing Purpose}

Designing is a very important stage in making a tool, by plotting the components used so the tool will be made can work in accordance with expectation. So the purpose of designing a CNC drawing machine on this acrylic board is to be able to produce the drawing design we want to accelerate and to simplify the process of making the drawing design on the acryilic board.

In command to get satisfactory results then first we have to make good planning [11-46]. In this planning should be considered the characteristics of the components in command to facilitate the process.

The planning can be divided into some steps, namely :

a. System desigining

b. Mechanic desigining

In addition, with this design is the completion phase of the Final Report, implemented systematically and interrelated so obtained the expected tools..

\section{System Designing}

In designing the system tool that will be built is the Design of Computer Numerically Controlled Machine for Drawing Text On Arduino-Based Acrylic Board. The design of this tool is made with the help of block diagram for the whole system, where the block diagram is one of the most important part in the design of a tool, making block diagram is the first step done by the author before continuing to next step.Figure 10 is a block diagram that will be used on the tool to be designed.

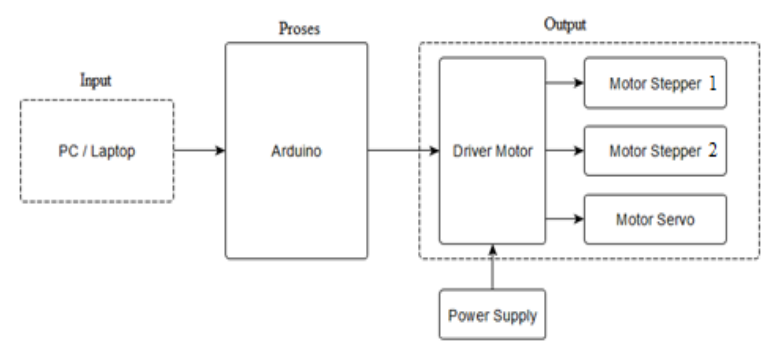

Fig. 10 CNC block diagram

Description of diagram block as shown in figure 10 :

1. Pc (Personal Computer) is an input that serves to transmit G-Code information that includes $\mathrm{x}, \mathrm{y}, \mathrm{z}$ data coordinate and movement data command then these data are transmitted by Pc with ASCII format via USB port.

2. Arduino microcontroller serves the translation of encompassing G-Code information data (coordinates $\mathrm{x}$, $\mathrm{y}, \mathrm{z}$ ) into realization of movement that will be run by motor.

3. Motor Driver serves as a circuit to control and to run the motor.

4. The motor serves to execute commands that will be sent by the microcontroller into the realization of movement, among others, motor stepper 1 will receive $\mathrm{x}$ coordinate data then moto stepper 2 will receive y coordinate data and servo motor will receive $\mathrm{z}$ coordinate data.

5. Power supply is one additional power used to run the motor

6. To control CNC drawing machine by giving a command or instruction in the form of serial data sent by the computer, consisting of instruction or command and G-code data in the form of numeric numbers. G-code data is data information which contain $\mathrm{X}$ axis, $\mathrm{Y}, \mathrm{Z}$ which are numeric number and must be done by machine.

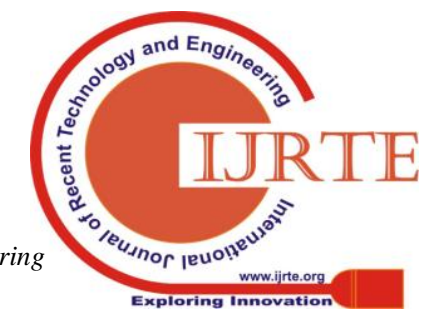




\section{Mechanic Designing}

Mechanical designing is the process of forming, cutting, installation of components such as the formation of mechanical design, cutting board plywood, drilling, and installation as sahft, bearing box, timming belt, stepper motor, and servo motor. After all is completed in the preparation of the final process is the installation of components in accordance with the design made.

\section{Components and Materials}

In making the right component selection circuit is very important. But before selecting component, there are several steps that can be done to get the right components.

1. The layout of components, in determining the location of the components must be designed first, so that it can be installed properly and correctlly later.

2. Learn circuit to be created

3. Learn to be used component characteristic

4. Then prepare the components, materials and tools that will be used in the process of making mechanics. The names of components, materials and tools can be seen in table 3 and table 4.

Table. 3 Component name

\begin{tabular}{clc}
\hline & \multicolumn{1}{|c}{ Component name } & Quantity \\
\hline 1 & Arduino Uno Microcontroler & 1 \\
2 & Adafruit Motor Driver & 1 \\
3 & Motor Stepper Nema 16 & 2 \\
4 & Motor servo & 1 \\
5 & Travo & 1 \\
6 & Dioda 4002 & 4 \\
7 & 220 uF and 100 uF capacitor & 1 \\
8 & IC L7815 & 1 \\
9 & 8mm As shaft & 4 \\
10 & 6mm As shaft & 2 \\
11 & 8mm Bearing Box & 6 \\
12 & Timming Belt & 3 Meter \\
13 & Pulley & 2 \\
14 & 8mm Bracket & 4 \\
\hline
\end{tabular}

Table. 4 Material and tool name

\begin{tabular}{cllc}
\hline No & Material and Tool Name & Quantity \\
\hline 1 & PCB Board with Hole & Sufficiently \\
2 & Solder & 1 \\
3 & Lead & Sufficiently \\
4 & PCB Leg & 4 \\
\hline
\end{tabular}

In the mechanical design the main thing is to design the place of the circuits in accordance with the size and in order to be protected and look neat. In the designing process this mechanical construction tools and materials are required as follows:

1. Saw

2. Plywood $10 \mathrm{~mm}$

3. Ruler

4. Drill

5. Bolts and Nuts

6. Screwdriver

7. Wood glue

To create a mechanical construction it can be done several stages namely as

1. Measuring stage
All materials to be cut like plywood in the measurement using a ruler according to predetermined size on each side and surface.

2. Cutting stage

Measure materials were cut according to determined size

3. Drilling stage

At this stage is done very carefully so that the distance between the holes with each other must be same and the distance between a hole to another hole must be same.

4. Combining stage

The next stage is combining which has been cut and perforated to form as we design.

5. Assembly stage

The last stage is to install and manage the components, tools and materials used.

The following is the overall mechanical design of Computer Numerically Controlled Design for Machine Drawing on Arduino-Based Acyilic Board, it can be seen in Figure 11.

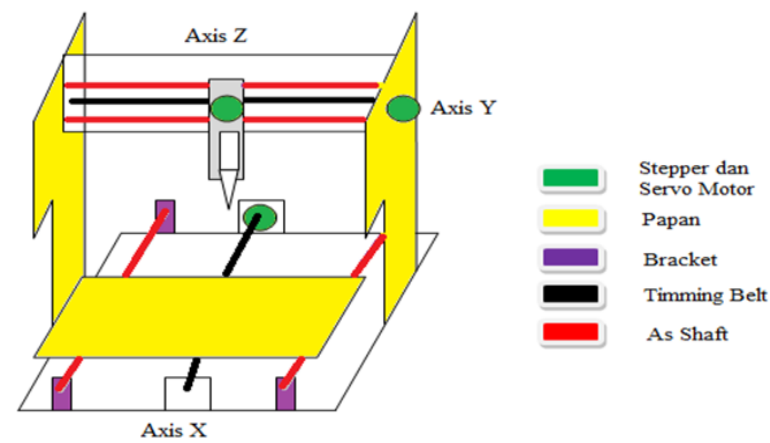

Fig. 11Design of Computer Numerically Controlled machine for drawing text on the arduino-based acrylic board

Tool Designing Stage

\section{Arduino Uno Microcontroler Circuit}

This circuit is the main control of all operating processes of Computer Numerically Controlled tools. This circuit will receive input from the program that we will create and then forward it to the output that will run the tool. The Arduino Uno circuit schematic can be seen in figure 12 . 


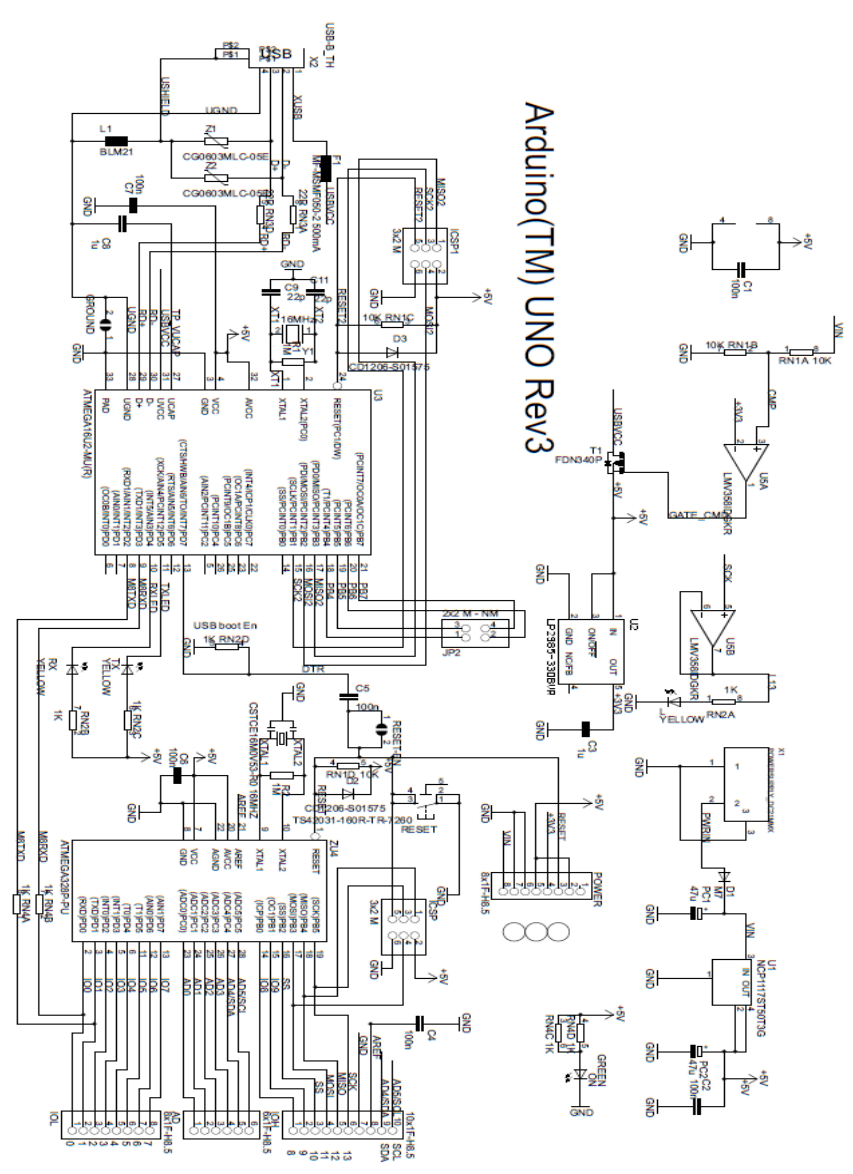

烦照

\section{Fig. 12 Arduino Uno Schematic}

\section{Adafruit Motor Driver Circuit}

Figure 13 is a circuit of motor drivers that works to run the motor stepper and motor servo.

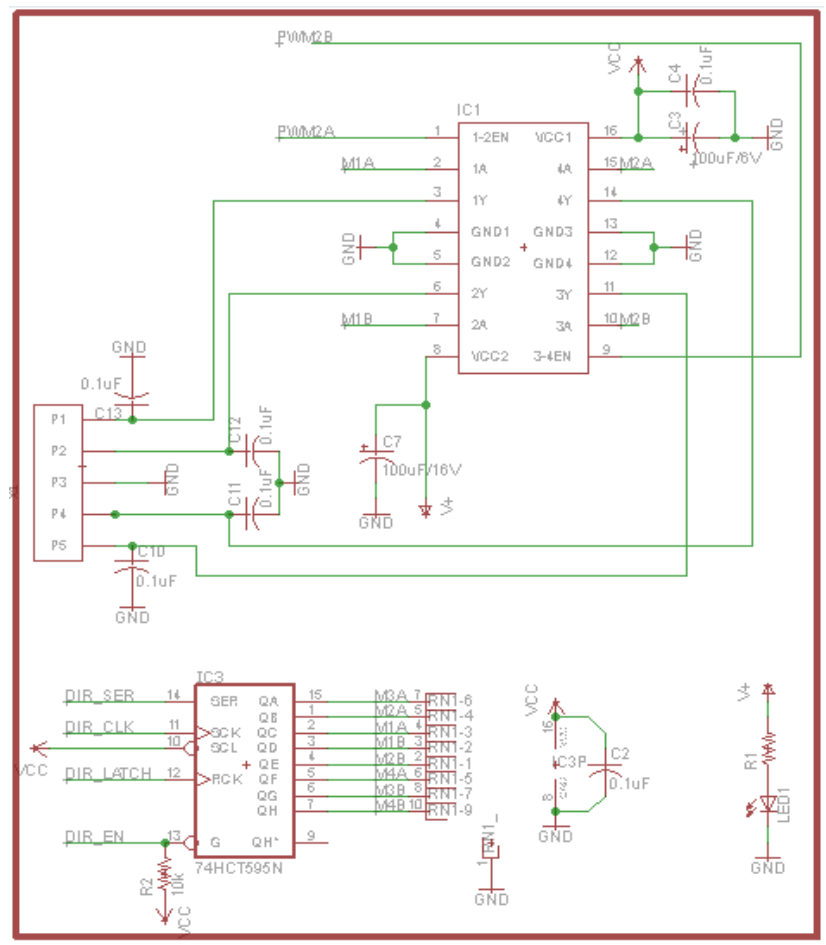

Fig. 13 Adafruit Motor Driver Schematic

\section{Circuit Working Ways}

There are several stages that a person must do before using CNC machine, firstly recognize some coordinate systems that exist on CNC machines, namely:

a. The coordinate system consists of $\mathrm{X}, \mathrm{Y}$ and $\mathrm{Z}$ axis where each Axis has a certain function consisting of absolute (absolute) coordinates and relative (incremental) coordinates

b. Coordinate system (polar coordinate), which consists of absolute (absolute) coordinates and relative (incremental) coordinates. Next we can determine the coordinate system that will be used in programming.

Absolute programming is a programming which in determining coordinate point always refers to the point of zero workpiece. The position of the point in the workpiece always starts from the point of zero as a reference. As a workplace reference point the location of zero point is determined based on the shape of the workpiece and the effectiveness of the program to be created. Zero point determination refers to the zero point of the workpiece. In complex workpiece programming, through certain G-code codes the zero point of the workpiece can be moved as needed to facilitate programming and to avoid errors. Absolute programming is also known as the absolute programming system, in which the movement of the drawing tool of the text refers to the zero point of the workpiece. The advantage of this system in case of programming error only affect the point in question, making it easier to make corrections.

Determination of text tool drawing movement from point one to the next point refers to the last stop point of the text drawing tool. Determination of step by step.

The disadvantage of this programming system, if there is an error in the determination of the coordinate point, then the result of the text drawing will not be not in accordance with the expectation.

Cartesian coordinate system for the motion of drawing text on three major $\mathrm{X}, \mathrm{Y}, \mathrm{Z}$ axis. When the movement of workpiece is given a symbol $\left(\mathrm{X}^{\prime}, \mathrm{Y}^{\prime}, \mathrm{Z}^{\prime}\right)$. The application of the axis symbol on the CNC machine draws text following certain rules, starting with the $\mathrm{Z}$ axis, followed by the $\mathrm{X}$ axis and finally the $\mathrm{Y}$ axis.

The motors used on the $\mathrm{CNC}$ axis machines are motor stepper and motor servo The motor stepper is an electromechanical device that works by converting electronic pulses into discrete mechanical motions. Motor stepper moves based on the order of pulses given to the motor. Therefore, to drive the motor stepper required a motor stepper controller that generates periodic pulses.

The rotation angle of motor is proportional to the input pulse so it is more manageable, so the motor shaft can be adjusted to rotate with some degrees

a. The motor can directly provide full torque at the start of moving

b. The position and movement of repetitions can be determined precisely

c. The motor has a very good response to start, stop and turn (rotation)

Axis motor is the driver motor for each $\mathrm{CNC}$ axis machine.

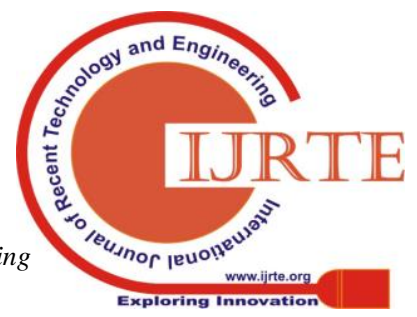


Both the $\mathrm{X}$ axis, $\mathrm{Y} \& \mathrm{Z}$, each of them has their own axis motors. Axis motors in general are a motor stepper and motor servo. The motor will move at the point or location of $\mathrm{X}, \mathrm{Y}, \mathrm{Z}$ which are instructed by computer, to make it easier to create drawing, we can use inkscape program to create the design, then interpreted from the image to the understandable $\mathrm{G}$-code language and implemented by $\mathrm{CNC}$ machines.

\section{RESULTS AND DISCUSSION}

Testing the tool is an important stage in tool making. Because at this stage will determine whether or not the tool is made in accordance with its usefulness so that it can be used in accordance with its needs as a drawing machine on the acrylic board.

Measurement and test in the designed circuit in the previous chapter was done by paying attention to measurement point (T). Then the next thing to do is to analyze the results of the test so that we can know the advantages and disadvantages of the tool made.

\section{The Purpose of Measurement}

The purposes of created tool measurement are :

1. Studying the incoming and outgoing voltages at each test point.

2. Observing and knowing whether the designed tool has been designed in accordance with the plan made.

3. Collecting data as a comparison between analyzing theoretically with practice that has been done.

4. This measurement is also done to determine if there is error in the circuit.

\section{Measurement Test}

Before measuring at the test point, it should prepare a multimeter measuring instrument that can measure the amount of obtained voltage. The measurement and analysis steps writers done namely as follows:

1. Turn on the circuit power and plug it into a 15 volt voltage source.

2. Prepare the multimeter in VDC state to measure the voltage

3. Calibrate the multimeter used first to measure and to obtain accurate results.

4. Determine the test point of each circuit.

5. Connect the input point to the positive pole on the multimeter and ground to the negarive pole in the multimeter.

6. Record the measurement results from each test point.

\section{Measurement Result}

\section{Measurement Result of X Axis motor stepper circuit}

In this tool there is a circuit of motor driver that serves to distribute electrical nergy in need for stepper motor movement based on information / command given by microcontroller. To be tested this time is the voltage that enter to motor stepper motor when motor stepper is given state of the load. Figure 14 is a stepper motor driver sequence for axis $\mathrm{x}$ that is acrylic drive from top to bottom.

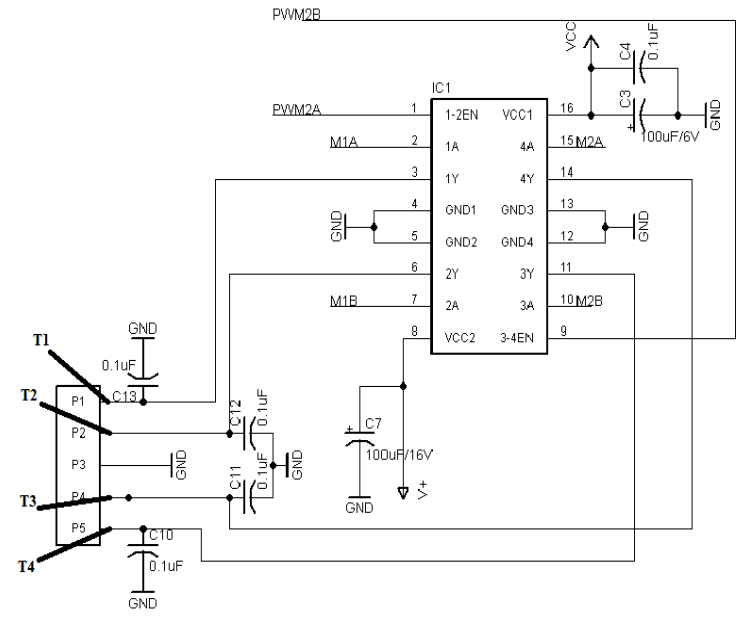

Fig. 14Motor Driver Stepper X Axis Circuit From the circuit there are 4 test points, Table 5 is the result of measurement.

Table. 5 The Result of Motor Stepper for X Axis measurement

\begin{tabular}{c|c|c|c}
\hline $\begin{array}{c}\text { Motor } \\
\text { Stepper } \\
\text { Voltage } \\
(\mathrm{T} 1)\end{array}$ & $\begin{array}{c}\text { Motor } \\
\text { Stepper } \\
\text { Voltage (T2) }\end{array}$ & $\begin{array}{c}\text { Motor } \\
\text { Stepper } \\
\text { Voltage } \\
(\mathrm{T} 3)\end{array}$ & $\begin{array}{c}\text { Motor } \\
\text { Stepper } \\
\text { Voltage (T4) }\end{array}$ \\
\hline $7,2 \mathrm{v}$ & $0 \mathrm{v}$ & $0 \mathrm{v}$ & $0 \mathrm{v}$ \\
$0 \mathrm{v}$ & $2 \mathrm{v}$ & $0 \mathrm{v}$ & $0 \mathrm{v}$ \\
$0 \mathrm{v}$ & $0 \mathrm{v}$ & $2,8 \mathrm{v}$ & $0 \mathrm{v}$ \\
$0 \mathrm{v}$ & $0 \mathrm{v}$ & $0 \mathrm{v}$ & $2,8 \mathrm{v}$ \\
\hline
\end{tabular}

\section{The measurement result of Y Axis motor stepper}

Figure 15 is Figure of motor driver stepper circuit for $\mathrm{Y}$ axis that is Pen drive from right to left or vice versa.

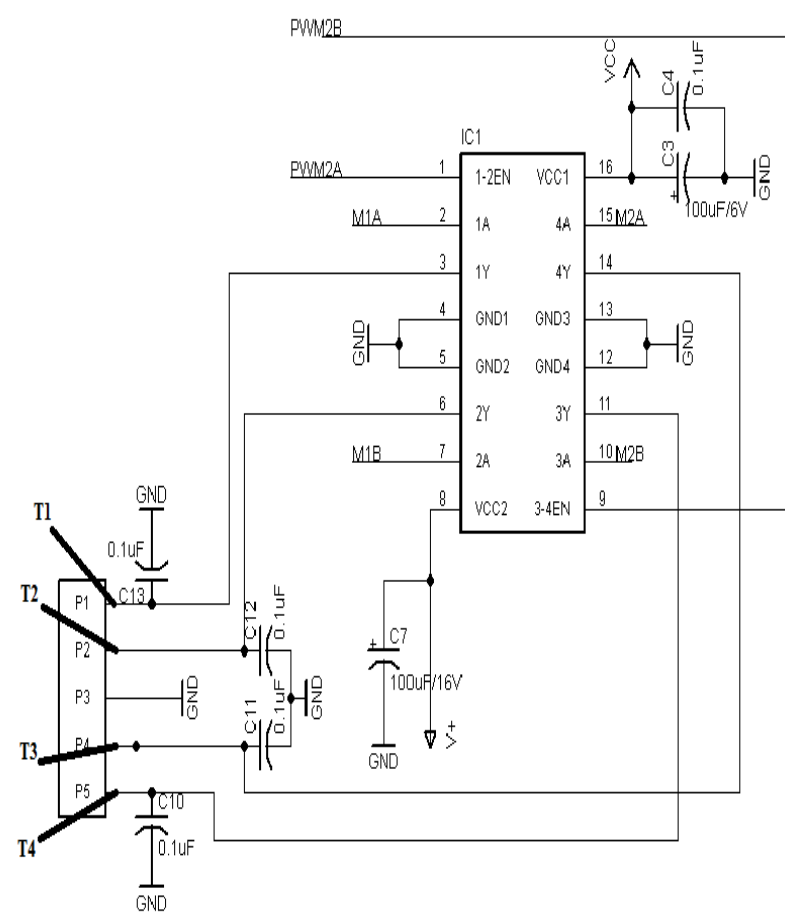

Fig. 15 Y Axis motor stepper driver circuit

From that circuit there are 4 test points. Table 6 is the measurement result 
Table. 6The Result of Motor Stepper for X Axis measurement

\begin{tabular}{c|c|c|c}
\hline $\begin{array}{c}\text { Motor } \\
\text { Stepper } \\
\text { Voltage } \\
(\mathrm{T} 1)\end{array}$ & $\begin{array}{c}\text { Motor } \\
\text { Stepper } \\
\text { Voltage (T2) }\end{array}$ & $\begin{array}{c}\text { Motor } \\
\text { Stepper } \\
\text { Voltage } \\
\text { (T3) }\end{array}$ & $\begin{array}{c}\text { Motor } \\
\text { Stepper } \\
\text { Voltage } \\
(\mathrm{T} 4)\end{array}$ \\
\hline $4 \mathrm{v}$ & $0 \mathrm{v}$ & $0 \mathrm{v}$ & $0 \mathrm{v}$ \\
$0 \mathrm{v}$ & $6 \mathrm{v}$ & $0 \mathrm{v}$ & $0 \mathrm{v}$ \\
$0 \mathrm{v}$ & $0 \mathrm{v}$ & $4 \mathrm{v}$ & $0 \mathrm{v}$ \\
$0 \mathrm{v}$ & $0 \mathrm{v}$ & $0 \mathrm{v}$ & \\
\hline
\end{tabular}

Measurement result of $\mathbf{Z}$ Axis motor servo circuit

Figure 16 is driver motor servo for $\mathrm{Z}$ axis that is pen driver from up to down or vice versa
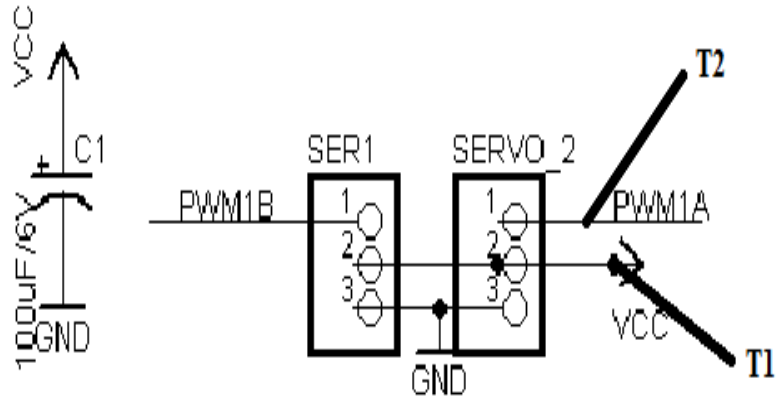

Fig. 16 Motor Driver Servo Axis Z circuit

From that current there are 2 testing ponts. Tabel 7 shows the measurement result.

Table.7 Measurement result of Motor Servo for $\mathbf{Z}$ Axis

\begin{tabular}{c|c}
\hline Trial & Motor Servo voltage (T1) VCC \\
\hline 1 & $5 \mathrm{v}$ \\
2 & $5 \mathrm{v}$ \\
3 & $5 \mathrm{v}$ \\
Average & $5 \mathrm{v}$ \\
\hline
\end{tabular}

\subsection{Program Testing Result}

Text drawing creation is to generate G-code as computer commands machine part was done by using inkscape software. Stages that must be done are as follow:

1. Open Inkscapeprogram, it will appear as Figure 17.

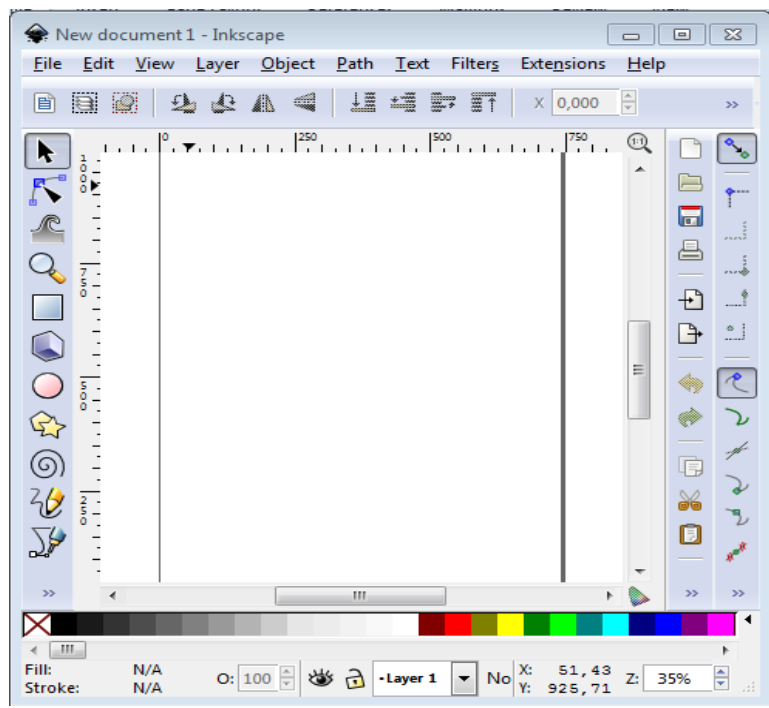

Fig. 17 Inkscape Program
2. Press Shift $+\mathrm{Ctrl}+\mathrm{D}$ to adjust the size of the layout used, and change the default units and units to $\mathrm{mm}$. Then create the text and Select Path and click Object to Path or click Shift $+\mathrm{Ctrl}+\mathrm{C}$, this serves to determine the coordinates between $\mathrm{X}, \mathrm{Y}$ and $\mathrm{Z}$. Then Save and select title with G-code and G-code data creation process completed .

3. Open CAMotic program, then open created CAMotic project using Inkscape program, such as Figure 18CAMotic project which has been created in inkscape. It can be run then just send the data to the microcontroller using the processing program.

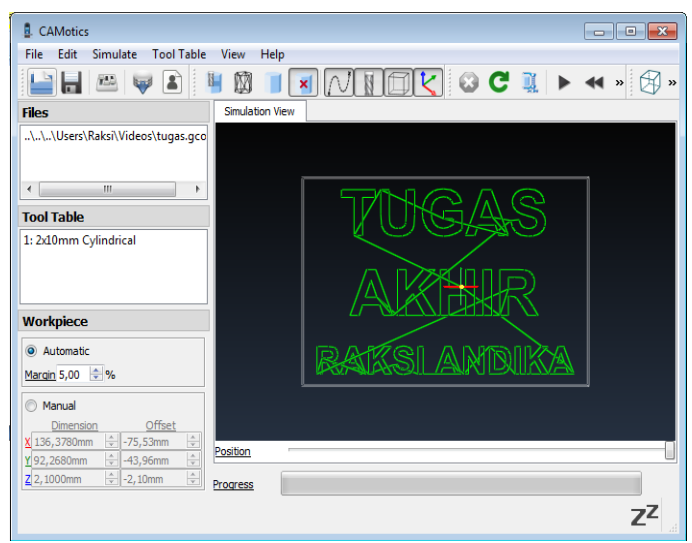

Fig. 18 CAMotic Project

4. Open processing program, Select File -> Click Open $\rightarrow$ Select gctrlgcode $\rightarrow$ click open. Click Run processing, it will display the program menu, there are various settings that can be seen in Figure 19.

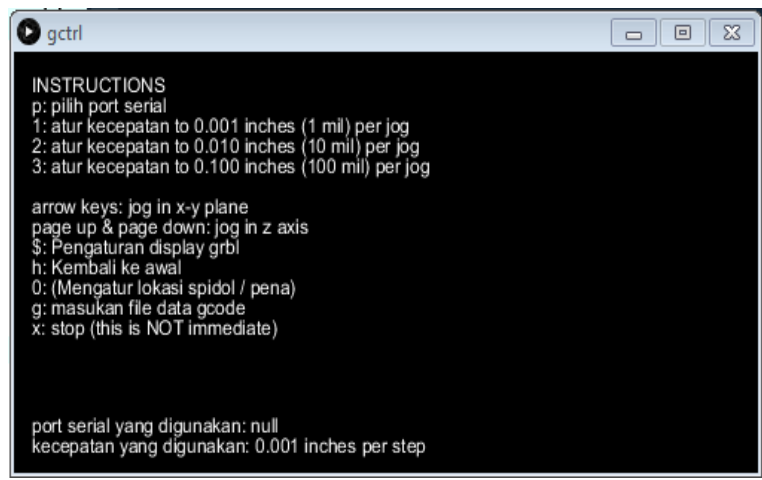

Fig. 19Gctrl Menu

5. Click $\mathrm{p}$ to select the serial port, 1,2,3 is used to set the speed, then select $g$ to enter G-code data and the program will automatically run according to the coordinates that have been made.

\section{Discussion}

The main part of controlling the motor is a motor driver that serves to control the performance of the motor in performing movement based on commands from the microcontroller. As the unit that has the main function to perform the code translation then the microcontroller must be connected with the output data from the computer via serial usb communication lines. 
First create a text drawing design using inkscape program, open inkscape program select file $\rightarrow$ document properties like Figure 20 below:

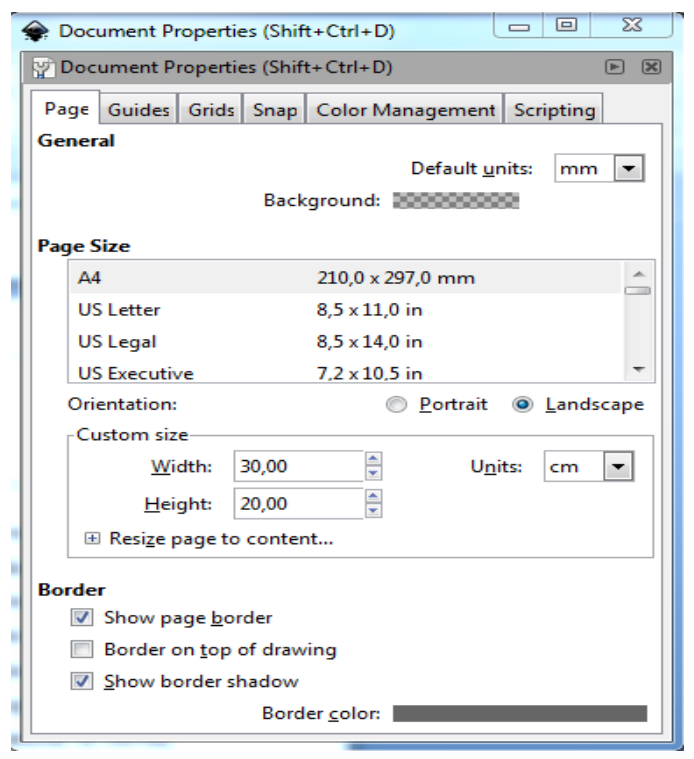

Fig.20 Document Properties Inkscape

Enter the text in the layout and then select Path $->$ click Object To Path it will show coordinate points such as Figure 21 where the inkscape program will read the edge of the object of the text that was created. so it can generate coordinate points that will be recognized by the $\mathrm{CNC}$ machine to draw the text on the acrylic board.

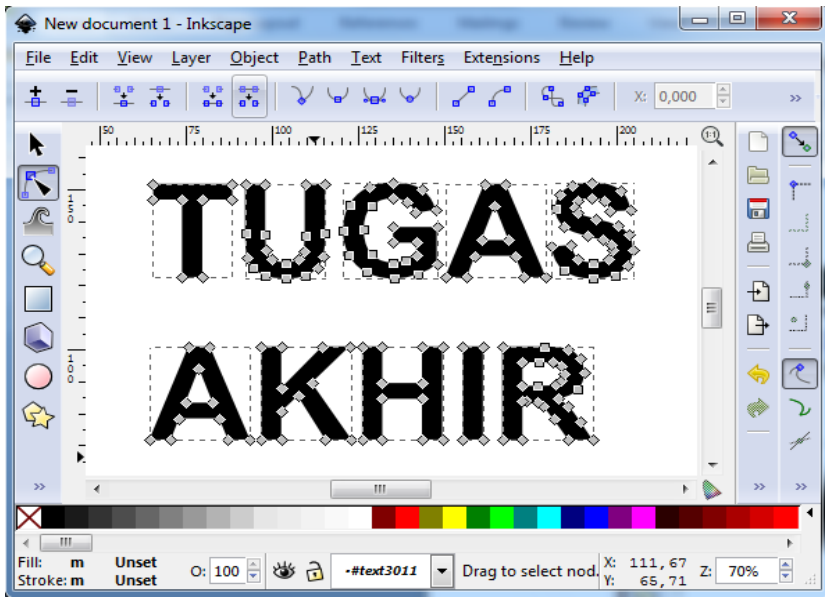

Fig. 21 Coordinaet points

Then save the g.code extension ., then the result will show the g.code extension file, as Figure 22 .gcode file that has been converted into coordinate $\mathrm{X}, \mathrm{Y}, \mathrm{Z}$ points.

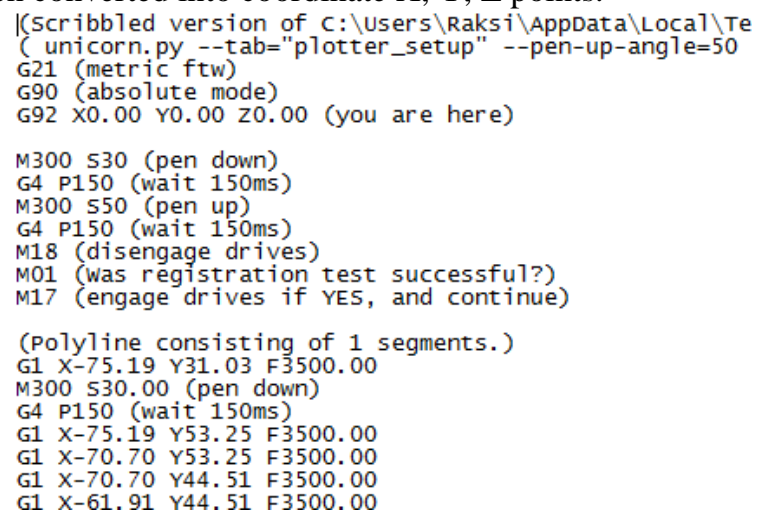

Fig. 22 G-Code coordinate
At the main program the calling of servo initialization and stepper while waiting for the interrupt done. so At $S$ ervoPWMInit function, TCCR register setting to get fast PWM mode and prescaler 64 for frequency of $50 \mathrm{~Hz}$. the ServoPWMGenerator is counter PWM with OCR register with pulse width input and calls the servoPWMGenerator with the conversion of degrees to pulse widths and sets the value of PORT used for Stepper, where the PWMGenerator servo program must specify the degree of PenUP (80 degrees) and PenDown (50 degrees ). then for motor stepper motion for step score entered and function of $\mathrm{X}$ move and $\mathrm{Y}$ move, it was done calling step function equal to desired input value $(\mathrm{mm})$ In this $\mathrm{Z}$ move, a ServoSetAngle call is made with the desired angle value. then stepper motion to form a design that is made according to coordinates.

To draw a slash, we need a trick to ge good results. The fact is, stepper motors are active at one time only one. So we can only move this $\mathrm{CNC}$ on the $\mathrm{X}$ or $\mathrm{Y}$ axis at a time. Therefore we move $\mathrm{X}$ or $\mathrm{Y}$ at different times in a short time. Therefore, every long slash, we turn it into small pieces called dividers. Figure $23 \mathrm{X}$ and $\mathrm{Y}$ segments with divider

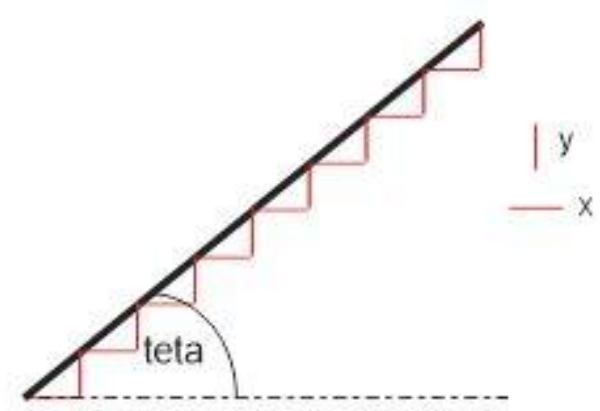

Fig. 23X and Y segment with Divider

By using the Divider segmentation function in the previous explanation, we can represent the $\mathrm{N}$-segment as a function of the Segmentation Divider by using the angle in the form of a function of I (serial number of side).

The position of the markers used wis determined using the servo used. The servo will rotate the marker, so it can stay away from the Acrylic board or attach it to the Acrylic board.

Therefore the drawing that has been tried is the Text drawing. Figure 24 shows the experimental results of the tool.

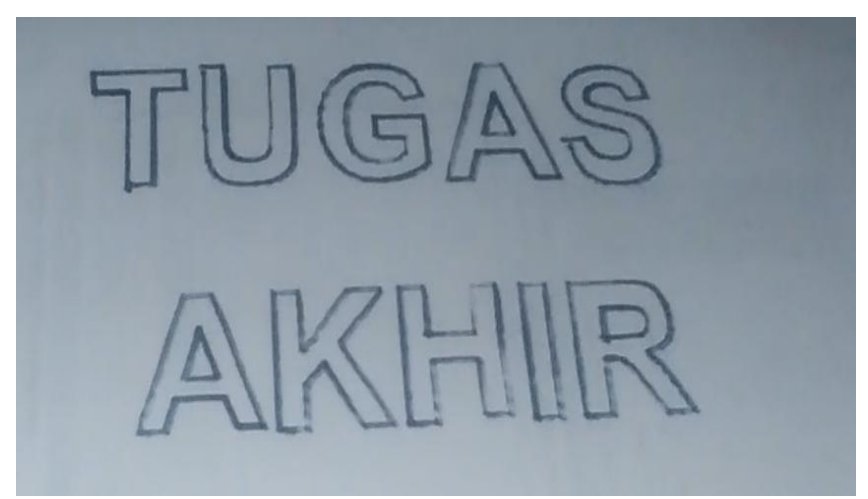

Fig. 24 Drawing result of CNC machine text

Published By:

Blue Eyes Intelligence Engineering \& Sciences Publication 


\section{CONCLUSION}

Based on the results of discussion and description of all data about the ways of chain working obtained some conclusions: 1) To control the text drawing tool it consisted of two stepper motors and one servo motor representing the respective functions of $x, y$, and $z$ coordinate axis. 2) To control the stepper motor we used 4 digital pins for switching step.Suggestion: 1) This tool can also be made into three dimensions to perform text drawing. 2) To improve the performance of the tools in the future, because this tool is only focused to draw the text is expected to not only draw the text but also can draw another shape.

\section{References}

1. Pandian, S.; Pandian, S.R. 2014. A Low Cost Build Your Own Three Axis CNC Mill Prototype, International Journal on Mechanical Engineering and Robotics (IJMER), v. 2, n. 1, pp. 2321-5747.

2. Prince, M.K.K.; Ansary, M.A.M.; Mondol, A.S. 2017. Implementation of Low Cost CNC Plotter using Spare Parts, International Journal of Engineering Trends and Technology (IJETT), v.43, n.6, pp. 333-339.

3. Kashikar, K.; Bhagat, R.; Nachare, A.; Shigwan, A.; Palinje, M. 2018. Smart Axibot. International Conference on Innovative and Advanced Technologies in Engineering, v.1, pp. 12-17.

4. Yang, H.B.; Yan, J. 2015. DXF File Identification with C\# for CNC Engraving Machine System, Intelligent Control and Automation, pp. 20-28.

5. Sutarman; Edihermawan, H.; Sarmidi. 2017. Computer Numerical Control (CNC) Milling and Turning for Machining Process in Xintai Indonesia, Quest Journals: Journal of Research in Mechanical Engineering, v.3, n.5, pp. 01-07.

6. Miftakul Amin, M.; AzelAjiNugratama, M.; Maseleno, A.; Huda, M.; Jasmi, K.A. 2018.Design of Cigarette Disposal Blower and Automatic Freshner using MQ-5 Sensor Based on ATMEGA 8535 Microcontroller,International Journal of Engineering and Technology(UAE), v. 7, no. 3, pp. 1108-1113

7. Sharan, R.V.; Onwubolu, G.C. 2013. Simulating the Arm Movements of a Stepper Motor Controlled Pick-and-Place Robot Using the Stepper Motor Model, International Journal of Advanced Science and Technology, v.6, pp. 59-66

8. Shah, N.M.; Arya, S. 2016. Simulation and Implementation of DC Servomotor Position Control: A Technological Review, International Journal of Advance Engineering and Research Development, v.3, n.2, pp. 124-129.

9. Laddha, N.R.; Thakare, A.P. 2013. A Review on Serial Communication by UART, International Journal of Advanced Research in Computer Science and Software Engineering, v.3, n.1, pp. 366-369.

10. Widodo, S.; Amin, M.M.; Sutrisman, A. 2018. The Design of the Monitoring Tools of Clean Air Condition and Dangerous Gas CO, $\mathrm{CO} 2 \mathrm{CH} 4$ in Chemical Laboratory by Using Fuzzy Logic Based On Microcontroller. E3S Web of Conferences, ICENIS 2017, pp. 1-4.

11. Manikandan, V., et al. "PRIVACY PRESERVING DATA MINING USING THRESHOLD BASED FUZZY CMEANS CLUSTERING." ICTACT Journal on Soft Computing 9.1 (2018).

12. Batri, K., and M. Sivaram. "Testing the impact of odd and even point crossover of genetic algorithm over the data fusion in information retrieval." European Journal of Scientific Research (2012).

13. Steffin Abraham, TanaLuciyaJoji, Sivaram M, D.Yuvaraj, "Enhancing Vehicle Safety With Drowsiness Detection Andcollision Avoidance" International Journal of Pure and Applied Mathematics, Volume 118 No. 22 2018, 921-927. https://acadpubl.eu/hub/2018-118 22/articles/22b/39.pdf

14. M. Sivaram, K. Batri, Amin Salih Mohammed and V. Porkodi, "Exploiting the Local Optima in Genetic Algorithm using Tabu Search", Indian Journal of Science and Technology, Vol 12(1), DOI: 10.17485/ijst/2018/v12i1/139577, January 2019.

15. Nithya, S., SundaraVadivel, P., Yuvaraj, D., Sivaram, M. "Intelligent based IoT smart city on traffic control system using raspberry Pi and robust waste management", Journal of Advanced Research in Dynamical and Control Systems, Pages: 765-770, 2018.

16. Porkodi, V., et al. "Survey on White-Box Attacks and Solutions." Asian Journal of Computer Science and Technology 7.3 (2018): 28-32.

17. Viswanathan, M., Sivaram, M., Yuvaraj, D., Mohammed, A.S. "Security and privacy protection in cloud computing", Journal of Advanced Research in Dynamical and Control Systems, Pages 1704-1710, 2018
18. Mohammed, Amin Salih, D. Yuvaraj, M. Sivaram, and V. Porkodi. "Detection And Removal Of Black Hole Attack In Mobile Ad Hoc Networks Using Grp Protocol." International Journal of Advanced Research in Computer Science 10, no. 6 (2018).

19. Sivaram, M., B. DurgaDevi, and J. Anne Steffi. "Steganography of two LSB bits." International Journal of Communications and Engineering 1.1 (2012): 2231-2307.

20. Dhivakar, B., et al. "Statistical Score Calculation of Information Retrieval Systems using Data Fusion Technique." Computer Science and Engineering 2.5 (2012): 43-5.

21. K.Mahalakshmi, M.Sivaram, K. ShanthaKumari, D.Yuvaraj, R.Keerthika, "Healthcare Visible Light Communication", International Journal of Pure and Applied Mathematics, Volume 118 No. 112018 , 345-348, https://acadpubl.eu/jsi/2018-118-10-11/articles/11/41.pdf.

22. Punidha, R.,Pavithra K, Swathika R, and Sivaram M, “ Preserving DDoS Attacks sing Node Blocking Algorithm.” International Journalof Pure and Applied Mathematics, Vol.119, o. 15, 2018, pp 633- 640. https://acadpubl.eu/hub/2018-119-15/3/473.pdf

23. Sivaram.M, Yuvaraj.D, Amin Salih Mohammed, Porkodi.V "Estimating the Secret Message in the Digital Image" International Journal of Computer Applications, 181(36):26-28, January 2019.

24. Punidha, R., avithra K, Swathika R, and Sivaram M, “Preserving DDoS Attacks sing Node Blocking Algorithm." International Journalof Pure and Applied Mathematics, Vol.119, o. 15, 2018, pp 633-640. https://acadpubl.eu/hub/2018-119-15/3/473.pdf

25. Sivaram, M., Yuvaraj, D., Porkodi, V., Manikandan, V. "Emergent news event detection from facebook using clustering" Journal of Advanced Research in Dynamical and Control Systems, Pages:1941-1947, 2018

26. Sivaram M, Batri K, " Odd and Even Point Crossover Based Tabu GA for Data Fusion in InformationRetrieval",http://hdl.handle.net/10603/389 10-Apr-2015.

27. Sivaram, M. "Odd And Even Point Crossover Based TabuGa For Data Fusion In Information Retrieval." (2014)

28. Malathi, N., and M.Sivaram. "An Enhanced Scheme to Pinpoint Malicious Behavior of Nodes InManet's." (2015).

29. Mohammed, A.S., Kareem, S.W., Al Azzawi, A.K., Sivaram, M. "Time series prediction using SRE-NAR and SRE-ADALINE", Journal of Advanced Research in Dynamical and Control Systems, Pages: 1716-1726, 2018.

30. Mohammed, SivaramYuvaraj Amin Salih, and V. Porkodi. "Estimating the Secret Message in the Digital Image." International Journal of Computer Applications 181.36 (2019): 26-28

31. Sivaram, M., et al. "Estimating the Secret Message in the Digital Image." International Journal of Computer Applications 975: 8887.

32. Sivaram, M., et al. "The Real Problem Through a Selection Making an Algorithm that Minimizes the Computational Complexity."

33. Obulatha-II-ME-CSE, Miss O. "Position Privacy Using LocX."

34. SRE-NAR, Time Series Prediction Using. "SRE-ADALINE."

35. Kousik, N. V., M. Sivaram, and S. Kalidass. "AUTONOMOUS GREEDY ROUTING IN WIRELESS SENSOR NETWORKS."

36. S, Deepa and M, Sivaram, Enabling Anonymous Endorsement in Clouds with Decentralized Access Control (June 2015). International Journal of Scientific Engineering and Applied Science (IJSEAS) - Volume-1, Issue-3, June 2015. Available at SSRN: https://ssrn.com/abstract $=3352128$

37. M, Sivaram, 4G and 5G Communication Networks Future Analysis (April 11, 2019). Available at SSRN: https://ssrn.com/abstract=

38. M, Sivaram and Sivaram, Porkodi and manikandan, V, Securing the Sensor Networks Along With Secured Routing Protocols for Data Transfer in Wireless Sensor Networks (OCTOBER 28, 2018). Available at SSRN: https://ssrn.com/abstract=

39. Sivaram, M., et al. "DETECTION OF ACCURATE FACIAL DETECTION USING HYBRID DEEP CONVOLUTIONAL RECURRENT NEURAL NETWORK." ICTACT Journal on Soft Computing 9.2 (2019).

40. R.Punidha, M.Sivaram, "Integer Wavelet Transform Based Approach For High Robustness Of Audio Signal Transmission", International Journal of Pure and Applied Mathematics, Volume 116 No. 232017 , 295-304, https://acadpubl.eu/jsi/2017-116-23-24/articles/23/40.pdf 
41. Sivaram M., Mohammed A.S., Yuvaraj D., Porkodi V., Manikandan V., Yuvaraj N. (2019) Advanced Expert System Using Particle Swarm Optimization Based Adaptive Network Based Fuzzy Inference System to Diagnose the Physical Constitution of Human Body. In: Somani A., Ramakrishna S., Chaudhary A., Choudhary C., Agarwal B. (eds) Emerging Technologies in Computer Engineering: Microservices in Big Data Analytics. ICETCE 2019. Communications in Computer and Information Science, vol 985. Springer, Singapore

42. Sivaram M. et al. (2019) Expert System in Determining the Quality of Superior Gourami Seed Using Forward Chaining-Based Websites. In: Somani A., Ramakrishna S., Chaudhary A., Choudhary C., Agarwal B. (eds) Emerging Technologies in Computer Engineering: Microservices in Big Data Analytics. ICETCE 2019. Communications in Computer and Information Science, vol 985. Springer, Singapore

43. Amin Salih Mohammed et al., "Analysis of Mobile IP Wireless Networks in 5G International", Journal of Advanced Trends in Computer Science and Engineering, 8(1.2), 2019, 39- 42

44. S. JeyaShobana et al., "FCCP - NS: A Fair Congestion Control Protocol with N-Sinks in Wireless Sensor Networks", International Journal of Advanced Trends in Computer Science and Engineering, 8(1.2), 2019, 43- 51

45. Sivaram, M., et al. "Retransmission DBTMA Protocol with Fast Retransmission Strategy to Improve the Performance of MANETs." IEEE Access (2019).

46. Yuvaraj D, Sivaram M, Karthikeyan B, and JihanAbdulazeez, "Shape, Color and Texture Based CBIR System Using Fuzzy Logic Classifier" CMC, vol.59, no.3, pp.729-739, 2019. 